J. Dairy Sci. 98:7899-7905

http://dx.doi.org/10.3168/jds.2015-9551

(c) American Dairy Science Association ${ }^{\circledR}, 2015$.

\title{
Short communication: Determination of the ability of Thymox to kill or inhibit various species of microorganisms associated with infectious causes of bovine lameness in vitro
}

\author{
Megan Kulow, ${ }^{* 1}$ Fahimeh Zibaee, ${ }^{*}$ Marianne Allard, $†$ and Dörte Döpfer* \\ *Department of Medical Sciences, School of Veterinary Medicine, University of Wisconsin, 2015 Linden Dr., Madison 53706 \\ †Laboratoire M2, 4005-A, rue de la Garlock, Sherbrooke, Québec, Canada J1L 1W9
}

\begin{abstract}
Infectious claw diseases continue to plague cattle in intensively managed husbandry systems. Poor foot hygiene and constant moist environments lead to the infection and spread of diseases such as digital dermatitis (hairy heel warts), interdigital dermatitis, and interdigital phlegmon (foot rot). Currently, copper sulfate and formalin are the most widely used disinfecting agents in bovine footbaths; however, the industry could benefit from more environmentally and worker friendly substitutes. This study determined the in vitro minimum inhibitory concentrations and minimum bactericidal concentrations of Thymox (Laboratoire M2, Sherbrooke, Québec, Canada) for a selection of microorganisms related to infectious bovine foot diseases. Thymox is a broad-spectrum agricultural disinfectant that is nontoxic, noncorrosive, and readily biodegradable. The values for minimum inhibitory concentration and minimum bactericidal concentration indicated that Thymox inhibited growth and killed the various species of microorganisms under study at much lower concentrations compared with the recommended working concentration of a $1 \%$ solution. Overall, the values found in this study of minimum inhibitory concentration and minimum bactericidal concentration of Thymox show its potential as an alternative antibacterial agent used in bovine footbaths; however, field trials are needed to determine its effectiveness for the control and prevention of infectious claw diseases.
\end{abstract}

Key words: bovine lameness, footbath disinfectant, digital dermatitis, interdigital phlegmon

\section{Short Communication}

The high incidences of lameness in dairy cows cause major animal welfare problems and have huge effects

\footnotetext{
Received March 9, 2015.

Accepted July 31, 2015.

${ }^{1}$ Corresponding author: mkulow@vetmed.wisc.edu
}

on overall herd productivity (Refaai et al., 2013). Lameness disorders are the third most important factor affecting production, after mastitis and reproductive disorders (USDA-NAHMS, 2009). Infectious claw diseases continue to affect cattle in intensively managed husbandry systems. Poor foot hygiene and constantly moist environments lead to the infection and spread of diseases such as digital dermatitis (DD; hairy heel warts), interdigital phlegmon (foot rot), and interdigital dermatitis (ID) with heel horn erosion (slurry heel) (Berry, 2001; Somers et al., 2005). Digital dermatitis was first documented in Italy in 1974 (Cheli and Mortellaro, 1974) and has since dramatically increased in prevalence, becoming one of the major causes of lameness in dairy cattle throughout the world (Wells et al., 1999; Yeruham et al., 2000; Manske et al., 2002; Somers et al., 2005; van Andel et al., 2012).

Bovine DD is a contagious and multifactorial disease that leads to painful, ulcerative lesions of the skin near the heel-horn border of the hoof. The etiology of this multifactorial claw disease is still not fully understood; however, strong evidence suggests Treponema subspecies are the main causative agents (Evans et al., 2008; Gomez et al., 2012). Other bacterial species that may play a role in the etiology of DD and have been isolated from lesions are Fusobacterium spp., Campylobacter spp., Peptococcus spp., Prevotella spp., Porphyromonas spp., and Dichelobacter nodosus (Dopfer et al., 1997; Ohya et al., 1999; Berry et al., 2010; Moe et al., 2010). Many of the microorganisms that are involved in DD are also involved in ID and foot rot (Berry, 2001). Specifically, Dichelobacter spp., Fusobacterium spp., and Porphyromonas spp. are causative agents in foot rot (Wani and Samanta, 2006). Dichelobacter nodosus has been implicated in the etiology of ID and may play a role in degrading the skin barrier, allowing for more invasive microbes to invade (Knappe-Poindecker et al., 2014).

Various forms of treatments have been used to prevent and control DD at either the group or cow level (Manske et al., 2002; Laven and Logue, 2006). Disin- 
fecting footbaths are a common management practice to control and prevent the spread of infectious diseases causing lameness, especially DD, within a herd. Copper sulfate and formalin are currently the most widely used antibacterial agents in footbaths; however, the industry could benefit from a more environmentally and worker friendly substitute (Speijers et al., 2010; Teixeira et al., 2010). Many alternative footbath products are available on the market; however, their effectiveness is often based largely on field experiences rather than randomized controlled trials. Because of the lack of data on many of the alternative footbath products, the manner in which they are used in the field is often anecdotal and their efficacy is often unproven (Thomsen, 2015). In vitro MIC and minimum bactericidal concentration (MBC) testing of alternative footbath products may aid in the development of an optimal footbath strategy for the control and prevention of infectious claw diseases.

The aim of this study was the in vitro determination of the MIC and MBC of Thymox (Laboratoire M2, Sherbrooke, Québec, Canada) for a selection of microorganisms related to infectious bovine foot diseases using a broth dilution method similar to that described by Hartshorn et al. (2013). A secondary aim was to compare the in vitro efficacy of copper sulfate (a commonly used antibacterial agent in the industry) and Thymox against the same selected microorganisms. Overall, we hypothesized that Thymox would be just as effective as copper sulfate at inhibiting growth and killing the microorganisms at or below the recommended working concentration.

Using a broth dilution method similar to that described by Hartshorn et al. (2013), the in vitro MIC and MBC values for Thymox and copper sulfate were established. Thymox is a broad-spectrum agricultural disinfectant with a footbath indication. The active ingredient of the proprietary formulation of Thymox is thymol ( $0.23 \%$ at the use dilution), a natural monoterpene phenol found, inter alia, in the oil of thyme. Thymox disinfectant is nontoxic (EPA 87742-1), noncorrosive (neutral $\mathrm{pH}$ ), and readily biodegradable (OCDE 301-E). Thymox is sold as a concentrate to be diluted at $1 \%$ in the footbath, with no special mixing or dissolving steps required.

The selection of representative microorganisms related to infectious bovine foot diseases used in the MIC-MBC assays included Campylobacter sputorum biovar faecalis (i.e., Campylobacter faecalis), Dichelobacter nodosus, Fusobacterium necrophorum, Fusobacterium nucleatum, Porphyromonas levii, Prevotella bivia, Prevotella denticola, Prevotella oralis, Treponema denticola-like, Treponema medium-like, and Treponema phagedenis-like. All of the microorganisms except the
Treponema species were obtained from ATCC (American Type Culture Collection, Manassas, VA). The ATCC did not offer any of the microorganisms directly isolated from bovine digital dermatitis lesions or any other source related to bovine infectious foot diseases. The selected microorganisms from the ATCC were chosen as representative of those that have been found to be isolated from infectious bovine hoof diseases.

Campylobacter faecalis (ATCC strain 33710) was isolated from sheep feces. Dichelobacter nodosus (ATCC strain 25549) and Fusobacterium necrophorum (ATCC strain 27852) were isolated from sheep with foot rot. No information was given regarding the isolation origin of Fusobacterium nucleatum (ATCC strain 23726). Porphyromonas levii (ATCC strain 29147) was isolated from rumen fluid. Prevotella bivia (ATCC strain 29303), Prevotella denticola (ATCC strain 33184), and Prevotella oralis (ATCC strain 33321) were of human origin. The 3 Treponema-like species are part of the Döpfer laboratory culture collection (School of Veterinary Medicine, University of Wisconsin, Madison) and were obtained from active DD lesions.

All of the microorganisms were cultured under anaerobic conditions $\left(70 \% \mathrm{~N}_{2}, 25 \% \mathrm{CO}_{2}\right.$, and $\left.5 \% \mathrm{H}_{2}\right)$ and incubated at $37^{\circ} \mathrm{C}$ expect for Campylobacter faecalis. Campylobacter faecalis was cultured in Brucella Albimi Broth (Anaerobe Systems, Morgan Hill, CA) in a microaerophilic atmosphere $\left(85 \% \mathrm{~N}_{2}, 10 \% \mathrm{CO}_{2}\right.$, and $5 \% \mathrm{O}_{2}$ ) at $37^{\circ} \mathrm{C}$. Dichelobacter nodosus was cultured in Tryptic Soy Broth (Becton, Dickinson, and Company, Sparks, MD) with 5\% sheep blood (Becton, Dickinson, and Company). Prevotella bivia was cultured in Reinforced Clostridial Broth (Anaerobe Systems). Fusobacterium necrophorum, Fusobacterium nucleatum, Porphyromonas levii, Prevotella denticola, and Prevotella oralis were cultured in Chopped Meat Medium (Anaerobe Systems). Treponema denticola-like, Treponema medium-like, and Treponema phagedenis-like were cultured in Oral Treponeme Enrichment Broth (Anaerobe Systems).

Briefly, MIC and MBC assays were performed in triplicate for Thymox and copper sulfate at 2 exposure times [minimal exposure (30 s) and 10-min exposure] using a broth dilution spot assay. At the start of each assay culture concentrations were determined using colony-forming unit plate counts from a 10-fold dilution series. For each assay, the stock disinfectants were diluted to recommended working concentrations: $1 \%$ Thymox and 5\% copper sulfate. The serial 2 -fold dilution series started at the working concentration and continued for 9 dilution steps to decreasing concentrations of the agents. Each assay contained negative controls (disinfectant in growth medium without microorganisms) and positive controls (microorganisms in 
growth medium without disinfectant). All spot assays and serial dilutions were cultured on anaerobic tryptic soy agar with $5 \%$ sheep blood plates (CDC formulation; Anaerobe Systems). Spot assay plates were checked routinely for growth, and MIC and MBC values were determined once new growth ceased to appear. This time period was variable (from 4 to $21 \mathrm{~d}$ ) due to the different growth rates of the microorganisms. One assay was conducted as described above for each microorganism expect for Fusobacterium nucleatum, in which the assay needed to be repeated a second time. An additional assay was performed to determine the MIC and $\mathrm{MBC}$ values for a microbial mix of the anaerobic microorganisms.

The final MIC and MBC values were calculated as an average of the triplicates in each assay for each microorganism, along with the standard deviations using Microsoft Excel (2007). The standard deviations of several MIC and MBC values were zero because values of the triplicate outcomes were the same. For descriptive comparison between Thymox and copper sulfate, the number of dilutions of the 2-fold dilution series to reach the MBC was determined. This was determined by counting the number of dilution steps (0 to 9 ) from the starting concentration (i.e., recommended working concentrations $1 \%$ solution for Thymox and 5\% solution for copper sulfate) until the MBC was reached.

The MIC and MBC values indicated that Thymox inhibited growth and killed the various species of microorganisms under study at much lower concentrations compared with the recommended working concentration of a $1 \%$ solution at both the minimal and 10-min exposure times (Figure 1). Copper sulfate also inhibited growth and killed the microorganisms under study at concentrations lower than the recommended working concentration of a $5 \%$ solution at both the minimal and 10-min exposure times for all but one of the selected microorganisms. Prevotella oralis was killed at the working concentration (MIC $=5 \%$ solution of copper sulfate) under minimal exposure time (Figure 2).

The concentration of the cultures of the selected microorganisms at the beginning of each assay varied from about $2.0 \times 10^{5} \mathrm{cfu} / \mathrm{mL}$ for Dichelobacter nodosus to as high as $1.5 \times 10^{10} \mathrm{cfu} / \mathrm{mL}$ for Porphyromonas levii (Table 1). Variable concentrations of starting cultures were observed due to different growth rates of

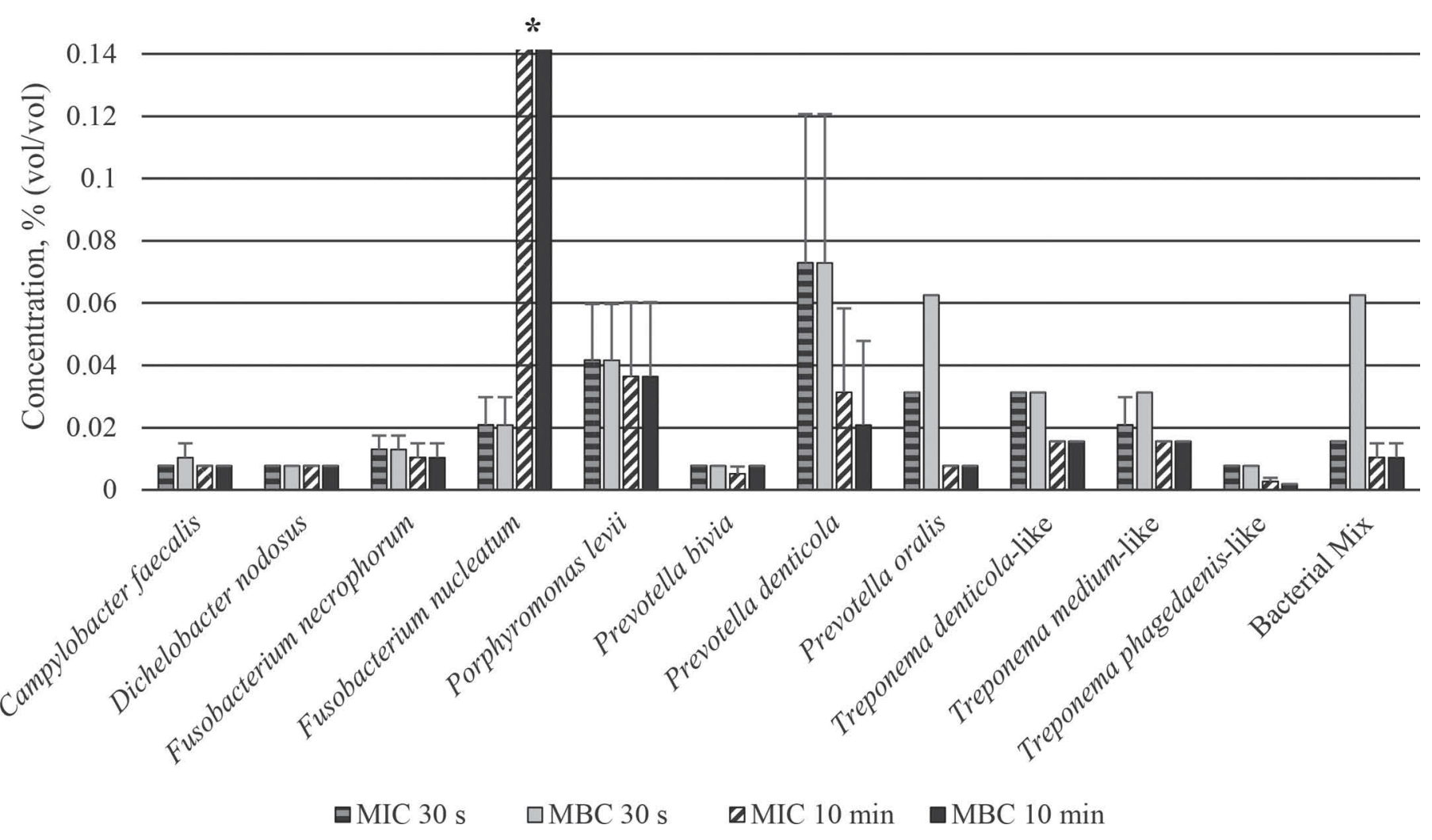

Figure 1. The in vitro MIC and minimum bactericidal concentration (MBC) values for Thymox (Laboratoire M2, Sherbrooke, Québec, Canada) reported as averages with standard deviations for each assay done in triplicate at a minimal exposure (30 s) and a 10-min exposure time. *The MIC and MBC values for Thymox for Fusobacterium nucleatum at the 10-min exposure time were larger than the readable scale of this figure, and the values are as follows: MIC and $\mathrm{MBC}=0.2083 \%$ with a $0.0721 \%$ standard deviation. 


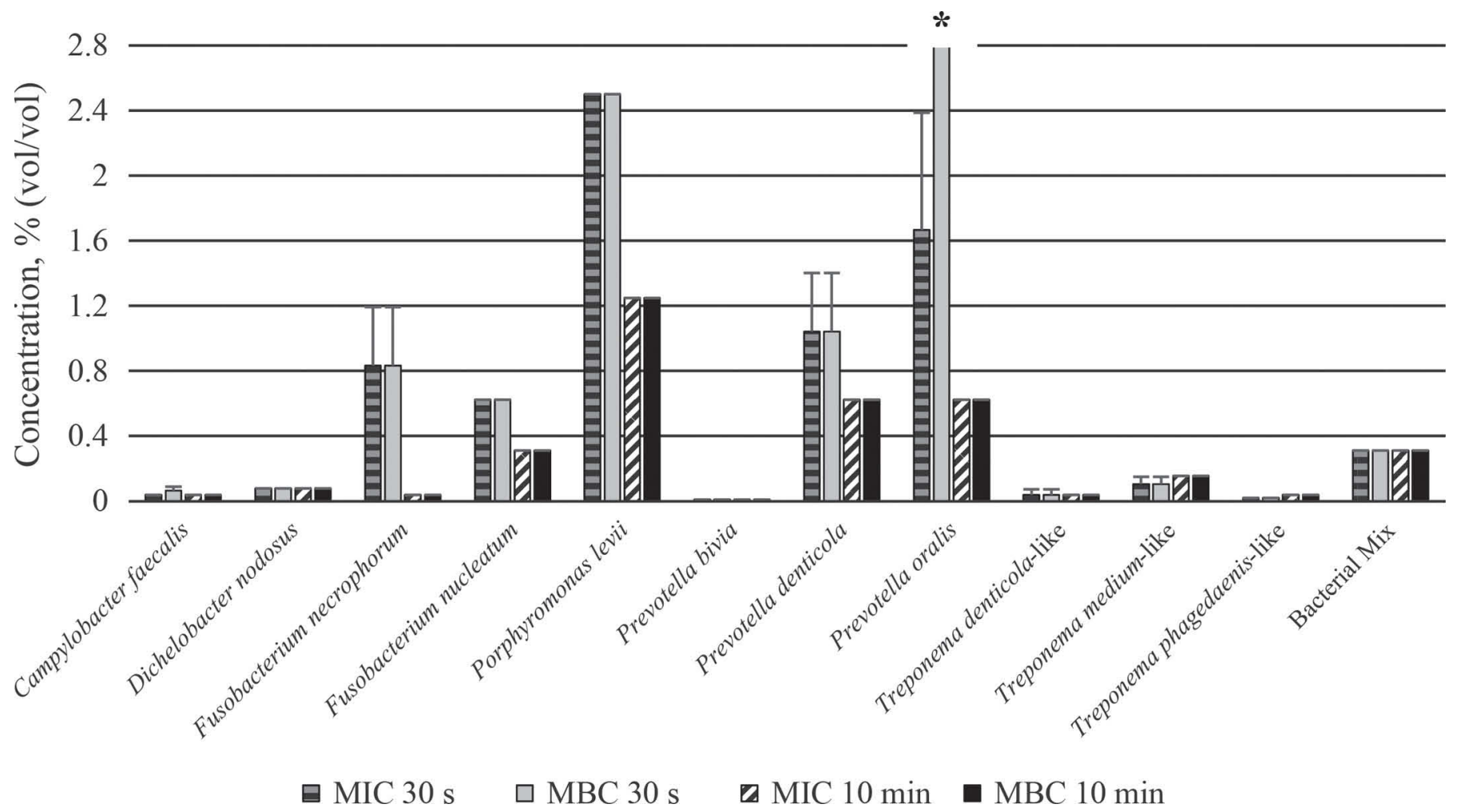

Figure 2. The in vitro MIC and minimum bactericidal concentration (MBC) values for copper sulfate reported as averages with standard deviations for each assay done in triplicate at a minimal exposure $(30 \mathrm{~s})$ and a 10 -min exposure time. *The MBC for copper sulfate for Prevotella oralis at the minimal exposure time $(30 \mathrm{~s})$ was larger than the readable scale of this figure, and the value is as follows: $\mathrm{MBC}=5 \%$.

the microorganisms. However, regardless of the starting culture concentration, for the majority of assays the microorganisms were killed at dilutions well below the recommended working concentrations (1\% Thymox and $5 \%$ copper sulfate) for either exposure time (Table 2 ). At the minimal exposure time, the number of dilution steps from the working concentrations to achieve the MBC ranged from 4 to 7 dilutions for Thymox, and for copper sulfate they ranged from 0 to 9 dilutions. At the 10-min exposure time, the number of dilution steps from the working concentrations to achieve the MBC ranged from 2 to 9 dilutions for Thymox and for copper sulfate. Overall, for each microorganism the $\mathrm{MBC}$ was reached with a more dilute solution after 10 min of exposure compared with the minimal exposure for both disinfectants. For unknown reasons, only $\mathrm{Fu}$ sobacterium nucleatum did not follow this trend; the MBC for the 10-min exposure was achieved at a less dilute solution compared with the minimal exposure for Thymox. When these results were first obtained for Fusobacterium nucleatum, the assay was repeated and resulted in the same trend.

Overall, at the minimal exposure time, Thymox killed 6 of the microorganisms (Dichelobacter nodosus, Fusobacterium necrophorum, Fusobacterium nucleatum, Por- phyromonas levii, Prevotella denticola, and Prevotella oralis) with more dilute solutions of the $1 \%$ working concentration compared with copper sulfate. However, the MBC for copper sulfate was reached with more dilute solutions for the Treponema species compared with Thymox. For the 3 species of treponemes, the MBC for Thymox was reached between 5 and 7 dilution steps from the $1 \%$ solution, and for copper sulfate the MBC

Table 1. Culture concentrations of each microorganism at the beginning of each MIC-MBC assay

\begin{tabular}{lc}
\hline Microorganism & $\begin{array}{c}\text { Concentration of cultures at } \\
\text { beginning of assay, cfu/mL }\end{array}$ \\
\hline Campylobacter faecalis & $2.2 \times 10^{5}$ \\
Dichelobacter nodosus & $1.9 \times 10^{5}$ \\
Fusobacterium necrophorum & $4.1 \times 10^{8}$ \\
Fusobacterium nucleatum & $2.5 \times 10^{9}$ \\
Porphyromonas levii & $1.5 \times 10^{10}$ \\
Prevotella bivia & $6.3 \times 10^{6}$ \\
Prevotella denticola & $5.2 \times 10^{8}$ \\
Prevotella oralis & $4.8 \times 10^{8}$ \\
Treponema denticola-like & $2.4 \times 10^{7}$ \\
Treponema medium-like & $1.4 \times 10^{7}$ \\
Treponema phagedenis-like & $1.0 \times 10^{6}$ \\
Bacterial mix & $5.6 \times 10^{7}$ \\
\hline
\end{tabular}

${ }^{1}$ Concentrations were determined using SPC. $\mathrm{MBC}=$ minimum bactericidal concentration. 
was reached between 7 and 8 dilution steps of the $5 \%$ solution. When all the anaerobic bacteria were mixed together in one assay (bacterial mix), the number of dilutions of the working concentration at which Thymox (1\%) and copper sulfate (5\%) killed the collection of microorganisms was the same for the minimal exposure time but varied for the 10-min exposure time. At the 10-min exposure time, the MBC for Thymox was achieved with a more dilute solution (6 dilution steps) compared with copper sulfate (4 dilution steps).

This study determined the in vitro MIC and MBC values of Thymox for 6 different representative species of microorganisms related to common infectious bovine claw diseases. Infectious diseases are often multifactorial in etiology and therefore require a variety of control measures to prevent outbreaks in intensively managed cattle husbandry systems. For example, DD involves environment, pathogens, hosts, and management factors. The pathogens in infectious foot diseases, such as DD, often involve multiple microorganisms requiring controls, preventions, and treatments that are effective against a whole range of microbes.

In this study, 6 different species of microorganisms were chosen based on the findings from previous research about their involvement in the pathogenesis of various bovine infectious causes of lameness, with a special emphasis on DD. The majority of research on DD supports Treponema spp. as the main bacterial causative agents in the disease, and 3 of the most commonly found strains are highlighted in the assays in this study: Treponema denticola-like, Treponema medium-like, and Treponema phagedenis-like (Choi et al., 1997; Dopfer et al., 1997; Evans et al., 2008). As the research community continues to determine the exact pathogenesis of DD, more and more research supports that DD is a polymicrobial disease (Santos et al., 2012; Krull et al., 2014; Zinicola et al., 2014). Several other bacterial species have been detected in active bovine DD lesions, and some include Campylobacter (Dopfer et al., 1997; Ohya et al., 1999), Dichelobacter (Rasmussen et al., 2012), Fusobacterium (Moe et al., 2010), Porphyromonas (Moe et al., 2010), and Bacteroides-like (Yano et al., 2010). Similarly to DD, foot rot and ID have polymicrobial involvement in their pathogenesis, and many of the microbes implicated in DD also play a role in these diseases.

Footbaths are a common management practice used on many dairies to control and prevent the spread of diseases like DD. Over the past 15 yr, a variety of antibacterial agents have been tested in controlled field studies to evaluate their efficacy in controlling infectious causes of lameness, particularly DD (Laven and Hunt, 2002; Holzhauer et al., 2008; Speijers et al., 2010; Logue et al., 2012; Smith et al., 2014). Copper sulfate was the most frequently used disinfecting agent in the studies and is also the most extensively used product in the field in North America. However, the disposal of spent footbaths containing copper sulfate has unintended consequences on the environment. Agricultural soils experience copper accumulation when footbaths are disposed of in wastewater lagoons used for irrigation purposes (Ippolito et al., 2010; Mateos-Naranjo et

Table 2. Number of dilutions below the recommended working concentrations to achieve complete kill of the microorganisms $(\mathrm{MBC})^{1}$

\begin{tabular}{|c|c|c|c|c|}
\hline \multirow[b]{3}{*}{ Microorganism } & \multicolumn{4}{|c|}{$\begin{array}{l}\text { Number of dilution steps }{ }^{2} \text { from the working } \\
\text { concentrations to achieve the MBC }\end{array}$} \\
\hline & \multicolumn{2}{|c|}{ Minimal exposure (30 s) } & \multicolumn{2}{|c|}{ 10-min exposure } \\
\hline & Thymox & Copper sulfate & Thymox & Copper sulfate \\
\hline Campylobacter faecalis & 6 & 7 & 7 & 7 \\
\hline Dichelobacter nodosus & 7 & 6 & 7 & 6 \\
\hline Fusobacterium necrophorum & 6 & 3 & 6 & 8 \\
\hline Fusobacterium nucleatum & 6 & 3 & 2 & 2 \\
\hline Porphyromonas levii & 5 & 1 & 5 & 2 \\
\hline Prevotella bivia & 7 & 9 & 7 & 9 \\
\hline Prevotella denticola & 4 & 2 & 6 & 3 \\
\hline Prevotella oralis & 4 & 0 & 7 & 3 \\
\hline Treponema denticola-like & 5 & 7 & 6 & 7 \\
\hline Treponema medium-like & 5 & 7 & 6 & 5 \\
\hline Treponema phagedenis-like & 7 & 8 & 9 & 7 \\
\hline Bacterial $\operatorname{mix}^{3}$ & 4 & 4 & 6 & 4 \\
\hline
\end{tabular}


al., 2013). Over extended periods, the accumulation of copper sulfate could slow OM decomposition and nutrient cycling in soil and reduce crop growth (Ippolito et al., 2011). Research continues to be conducted to find more environmentally friendly alternatives for footbath agents with similar efficacy and costs (Fiedler et al., 2013; Smith et al., 2014); however, a viable alternative to traditional disinfectants has not been clearly identified or implemented under the current management practices.

This study suggests that Thymox could be an alternative to commonly used disinfecting agents, such as copper sulfate and formalin, in bovine footbaths based on its ability to inhibit growth at the in vitro minimal exposure time at concentrations well below the recommended $1 \%$ working concentration. The in vitro minimal exposure time in this study is representative of the contact time of the foot with a footbath solution while the cow walks through a footbath and period time after while solution remains on the foot. In this study the ability of Thymox to inhibit growth and kill various species of bacteria was done using pure cultures, and it is reasonable to suspect that in a field situation, several factors that are difficult to reproduce in vitro might interfere with efficacy of Thymox, such as a shortened contact time of the disinfectant with the foot, the OM from manure in the footbath, the leg hygiene of the cow, or footbath design and management. However, given the low MIC and MBC values observed in the current study, one can speculate that a $1 \%$ solution should be effective at inhibiting growth and killing selected anaerobic microbes under field conditions. Plus, Thymox is biodegradable and nontoxic, making it a more environmentally and worker friendly alternative footbath solution at the recommended working concentration.

The implications of this type of in vitro testing of candidate chemicals, such as Thymox, for their effect of inhibiting and killing anaerobic bacteria involved in infectious claw diseases done in this study are that one knows the effects before testing them on live animals. Moreover, methods similar to those used in this study can be used to test the efficacy in the presence of OM in vitro (Hartshorn et al., 2013).

\section{ACKNOWLEDGMENTS}

The funding for this study was provided by Laboratoire M2 (Sherbrooke, Québec, Canada).

\section{REFERENCES}

Berry, S. L. 2001. Diseases of the digital soft tissues. Vet. Clin. North Am. Food Anim. Pract. 17:129.
Berry, S. L., D. H. Read, R. L. Walker, and T. R. Famula. 2010. Clinical, histologic, and bacteriologic findings in dairy cows with digital dermatitis (footwarts) one month after topical treatment with lincomycin hydrochloride or oxytetracycline hydrochloride. J. Am. Vet. Med. Assoc. 237:555-560.

Cheli, R., and C. Mortellaro. 1974. La dermatite digitale del bovino. Pages 208-213 in Proc. 8th Int. Meet. Diseases Cattle, Milan, Italy.

Choi, B. K., H. Nattermann, S. Grund, W. Haider, and U. B. Gobel. 1997. Spirochetes from digital dermatitis lesions in cattle are closely related to treponemes associated with human periodontitis. Int. J. Syst. Bacteriol. 47:175-181.

Dopfer, D., A. Koopmans, F. A. Meijer, I. Szakall, Y. H. Schukken, W. Klee, R. B. Bosma, J. L. Cornelisse, A. vanAsten, and A. terHuurne. 1997. Histological and bacteriological evaluation of digital dermatitis in cattle, with special reference to spirochaetes and Campylobacter faecalis. Vet. Rec. 140:620-623.

Evans, N. J., J. M. Brown, I. Demirkan, R. D. Murray, W. D. Vink, R. W. Blowey, C. A. Hart, and S. D. Carter. 2008. Three unique groups of spirochetes isolated from digital dermatitis lesions in UK cattle. Vet. Microbiol. 130:141-150.

Fiedler, A., K. Kraft, S. Reese, and J. Maierl. 2013. Influence of a new biocidal product on the prevalence of digital dermatitis in dairy cows. Tierarztl. Prax. Ausg. G Grosstiere Nutztiere 41:207-216.

Gomez, A., N. B. Cook, N. D. Bernardoni, J. Rieman, A. F. Dusick, R. Hartshorn, M. T. Socha, D. H. Read, and D. Dopfer. 2012. An experimental infection model to induce digital dermatitis infection in cattle. J. Dairy Sci. 95:1821-1830.

Hartshorn, R. E., E. C. Thomas, K. Anklam, M. G. Lopez-Benavides, M. Buchalova, T. C. Hemling, and D. Dopfer. 2013. Short communication: Minimum bactericidal concentration of disinfectants evaluated for bovine digital dermatitis-associated Treponema phagedenis-like spirochetes. J. Dairy Sci. 96:3034-3038.

Holzhauer, M., D. Döpfer, J. d. Boer, and G. v. Schaik. 2008. Effects of different intervention strategies on the incidence of papillomatous digital dermatitis in dairy cows. Vet. Rec. 162:41-46.

Ippolito, J. A., T. Ducey, and D. Tarkalson. 2010. Copper impacts on corn, soil extractability, and the soil bacterial community. Soil Sci. 175:586-592.

Ippolito, J. A., T. Ducey, and D. Tarkalson. 2011. Interactive effects of copper on alfalfa growth, soil copper, and soil bacteria. J. Agric. Sci. 3:11.

Knappe-Poindecker, M., M. Gilhuus, T. K. Jensen, S. Vatn, H. J. Jørgensen, and T. Fjeldaas. 2014. Cross-infection of virulent $D i$ chelobacter nodosus between sheep and co-grazing cattle. Vet. Microbiol. 170:375-382.

Krull, A. C., J. K. Shearer, P. J. Gorden, V. L. Cooper, G. J. Phillips, and P. J. Plummer. 2014. Deep sequencing analysis reveals temporal microbiota changes associated with development of bovine digital dermatitis. Infect. Immun. 82:3359-3373.

Laven, R. A., and H. Hunt. 2002. Evaluation of copper sulphate, formalin and peracetic acid in footbaths for the treatment of digital dermatitis in cattle. Vet. Rec. 151:144-146.

Laven, R. A., and D. N. Logue. 2006. Treatment strategies for digital dermatitis for the UK. Vet. J. 171:79-88.

Logue, D. N., T. Gibert, T. Parkin, S. Thomson, and D. J. Taylor. 2012. A field evaluation of a footbathing solution for the control of digital dermatitis in cattle. Vet. J. 193:664-668.

Manske, T., J. Hultgren, and C. Bergsten. 2002. Topical treatment of digital dermatitis associated with severe heel-horn erosion in a Swedish dairy herd. Prev. Vet. Med. 53:215-231.

Mateos-Naranjo, E., L. Andrades-Moreno, J. Cambrollé, and A. PerezMartin. 2013. Assessing the effect of copper on growth, copper accumulation and physiological responses of grazing species Atriplex halimus: Ecotoxicological implications. Ecotoxicol. Environ. Saf. 90:136-142.

Moe, K. K., T. Yano, K. Misumi, C. Kubota, K. Nibe, W. Yamazaki, M. Muguruma, and N. Misawa. 2010. Detection of antibodies against Fusobacterium necrophorum and Porphyromonas levii-like species in dairy cattle with papillomatous digital dermatitis. Microbiol. Immunol. 54:338-346. 
Ohya, T., H. Yamaguchi, Y. Nii, and H. Ito. 1999. Isolation of Campylobacter sputorum from lesions of papillomatous digital dermatitis in dairy cattle. Vet. Rec. 145:316-318.

Rasmussen, M., N. Capion, K. Klitgaard, T. Rogdo, T. Fjeldaas, M. Boye, and T. K. Jensen. 2012. Bovine digital dermatitis: Possible pathogenic consortium consisting of Dichelobacter nodosus and multiple Treponema species. Vet. Microbiol. 160:151-161.

Refaai, W., M. Van Aert, A. M. Abd El-Aal, A. E. Behery, and G. Opsomer. 2013. Infectious diseases causing lameness in cattle with a main emphasis on digital dermatitis (Mortellaro disease). Livest. Sci. 156:53-63.

Santos, T. M. A., R. V. Pereira, L. S. Caixeta, C. L. Guard, and R. C. Bicalho. 2012. Microbial diversity in bovine papillomatous digital dermatitis in Holstein dairy cows from upstate New York. FEMS Microbiol. Ecol. 79:518-529.

Smith, A. C., C. L. Wood, K. J. McQuerry, and J. M. Bewley. 2014. Effect of a tea tree oil and organic acid footbath solution on digital dermatitis in dairy cows. J. Dairy Sci. 97:2498-2501.

Somers, J. G. C. J., K. Frankena, E. N. Noordhuizen-Stassen, and J. H. M. Metz. 2005. Risk factors for digital dermatitis in dairy cows kept in cubicle houses in the Netherlands. Prev. Vet. Med. 71:11-21.

Speijers, M. H. M., L. G. Baird, G. A. Finney, J. McBride, D. J. Kilpatrick, D. N. Logue, and N. E. O'Connell. 2010. Effectiveness of different footbath solutions in the treatment of digital dermatitis in dairy cows. J. Dairy Sci. 93:5782-5791.

Teixeira, A. G. V., V. S. Machado, L. S. Caixeta, R. V. Pereira, and R. C. Bicalho. 2010. Efficacy of formalin, copper sulfate, and a commercial footbath product in the control of digital dermatitis. J. Dairy Sci. 93:3628-3634.

Thomsen, P. 2015. Short communication: Efficacy of copper sulfate hoof baths against digital dermatitis-Where is the evidence? J. Dairy Sci. 98:2539-2544.

USDA-NAHMS. 2009. Part IV: Changes in Dairy Cattle Health and Management Practices in the United States, 1996-2007. USDANatl. Anim. Health Monit. Syst., Fort Collins, CO.

van Andel, M., T. Rawdon, K. Thompson, and D. Vink. 2012. Review of recent bovine digital dermatitis-like lesions in cattle. Surveill. Wellingt. 39:9-13.

Wani, S. A., and I. Samanta. 2006. Current understanding of the aetiology and laboratory diagnosis of footrot. Vet. J. 171:421-428.

Wells, S. J., L. P. Garber, and B. A. Wagner. 1999. Papillomatous digital dermatitis and associated risk factors in US dairy herds. Prev. Vet. Med. 38:11-24.

Yano, T., K. K. Moe, K. Yamazaki, T. Ooka, T. Hayashi, and N. Misawa. 2010. Identification of candidate pathogens of papillomatous digital dermatitis in dairy cattle from quantitative $16 \mathrm{~S}$ rRNA clonal analysis. Vet. Microbiol. 143:352-362.

Yeruham, I., S. Friedman, D. Elad, and S. Perl. 2000. Association between milk production, somatic cell count and bacterial dermatoses in three dairy cattle herds. Aust. Vet. J. 78:250-253.

Zinicola, M., F. Lima, C. L. Guard, V. S. Machado, M. Gomez, and D. Dopfer. 2014. Altered microbiomes of bovine digital dermatitis lesions, and the gut as a pathogen reservoir. PLoS One 10:e0120504. 